Rabaska

Revue d'ethnologie de l'Amérique française

GUILCHER, JEAN-MICHEL. Danse traditionnelle et anciens

milieux ruraux français. Tradition, histoire, société. Paris, L'Harmattan, " Ethnomusicologie et anthropologie musicale de l'espace français », 2009, 318 p. ISBN 978-2-296-08258-8

\title{
Pierre Chartrand
}

Volume 11, 2013

URI : https://id.erudit.org/iderudit/1018538ar

DOI : https://doi.org/10.7202/1018538ar

Aller au sommaire du numéro

Éditeur(s)

Société québécoise d'ethnologie

ISSN

1703-7433 (imprimé)

1916-7350 (numérique)

Découvrir la revue

Citer ce compte rendu

Chartrand, P. (2013). Compte rendu de [GUILCHER, JEAN-Michel. Danse traditionnelle et anciens milieux ruraux français. Tradition, histoire, société. Paris, L'Harmattan, « Ethnomusicologie et anthropologie musicale de l'espace français ", 2009, 318 p. ISBN 978-2-296-08258-8]. Rabaska, 11, 210-213.

https://doi.org/10.7202/1018538ar d'utilisation que vous pouvez consulter en ligne. 
recherches empiriques et sur des documents de toute première main, comme le révèle la riche bibliographie en fin de volume.

Cet ouvrage de plus de 300 pages est encore abondamment et richement illustré, en couleurs, et se présente dans le format adopté avec la publication des autres livres de la collection "Atlas historique du Québec », soit un livre de $23 \mathrm{~cm}$ par $31 \mathrm{~cm}$. Au début de chaque chapitre, les auteurs ont eu l'excellente idée de présenter une carte géographique détaillée des frontières du territoire selon les périodes et n'ont pas lésiné sur les cartes, tableaux, graphiques, photos, de manière à illustrer davantage leurs propos ou ceux de leurs collaborateurs et collaboratrices. Quelques fautes ont toutefois échappé aux correcteurs, qu'il faudra corriger lors de la deuxième édition, en format plus pratique toutefois.

Il faudra tenir compte désormais de cet ouvrage, qui facilitera les recherches à venir sur la francophonie nord-américaine. Il faut féliciter les responsables qui ont eu la main heureuse dans le choix de leurs collaborateurs et collaboratrices. La Francophonie nord-américaine est un ouvrage incontournable, une véritable bible sur le sujet qui se lit comme un roman.

Aurélien Boivin

Université Laval, Québec

Guilcher, Jean-Michel. Danse traditionnelle et anciens milieux ruraux français. Tradition, histoire, société. Paris, L'Harmattan, « Ethnomusicologie et anthropologie musicale de l'espace français », 2009, 318 p. ISBN 978-2-296-08258-8.

En 2009 paraissait cet important ouvrage sur la danse traditionnelle par Jean-Michel Guilcher. Cela ne fit guère de vagues dans le milieu de la recherche en danse de ce côté-ci de l'Atlantique. Ce volume nous offre plus que des études de cas sur certaines traditions françaises, il nous livre en fait les réflexions profondes de l'auteur sur ce qu'est la danse traditionnelle, sur ses modes de conservation, d'évolution, de transformation... Et cela est d'un intérêt primordial pour quiconque veut aborder ce sujet, de quelque côté de l'Atlantique qu'il se trouve.

L'introduction est sans doute la partie qui m'a le plus touché. Non pas que l'essentiel de l'ouvrage ne rencontre pas nos attentes, bien au contraire. La pensée de Guilcher y est toujours fort éclairante, son expression toujours précise, élégante et avisée. C'est plutôt que l'auteur nous confie ses hésitations à écrire ce livre, qu'il nous parle de ses débuts dans la recherche en danse et de l'influence que son épouse a eu dans certaines de ses décisions. Il se pose ensuite la question essentielle de la conservation et 
du changement dans la société traditionnelle. Le fait que ses enquêtes aient débuté dès les années cinquante n'est évidemment pas étranger à l'intérêt de cet ouvrage, ses collectes lui ayant permis « de s'entretenir longuement avec des habitants des campagnes ayant eu vingt ans avant la fin du XIX ${ }^{\mathrm{e}}$ siècle » (p. 7). Il aborde longuement la question de la conservation et du changement dans la société traditionnelle. Sujet qui reviendra souvent au sein des chapitres suivants. Il en vient d'ailleurs à remettre en cause l'assomption trop commune suggérant que la société paysanne est essentiellement tournée vers la conservation de ses pratiques. Il dira de cette société rurale qu' " elle a montré dans le passé proche une aptitude tout aussi remarquable à donner forme à des états nouveaux... » (p. 10).

La question de la variation dans la danse est ainsi profondément analysée dans ce livre, et cela dans différents contextes. C'est sans doute un de ses grands mérites, surtout pour des chercheurs qui ne travaillent pas sur le répertoire français, mais qui pourront tirer profit de l'analyse et de la réflexion de Guilcher sur ces questions. On y voit par exemple que les sauts béarnais de la vallée de la Soule ont peu varié en comparaison de ceux de Basse-Navarre et du Béarn, ou que la Haute-Cornouaille fut plus « conservatrice » que les Côtes-d'Armor. Ces multiples exemples l'amènent à se questionner sur les variations possibles d'une époque à l'autre, entre des régions limitrophes, entre les interprétations des danseurs d'un même groupe social, dans une même danse. Il rappelle aussi que, « dans cet édifice fragile où tout est exposé à devenir autre, il y a au moins un élément qui offre plus de résistance, c'est cette organisation de l'ensemble que nous traduisons sur le papier par la formule rythmique des appuis. » (p. 10). C'est un enseignement incontournable à retenir du travail et de la méthode de Guilcher, qui nous rappelle également que la mémoire, telle que nous l'entendons souvent aujourd'hui, ne joue pas dans la société traditionnelle le rôle que nous lui donnons généralement. "L'homme n'a que faire de se souvenir, il lui suffit de s'abandonner à lui-même. Car plus que d'une connaissance acquise, il s'agit d'un geste qui fait partie de son être, et qu'il a pouvoir de vivre comme il vient, sans avoir aucunement besoin de s'y appliquer. » (p. 18). Ces trente premières pages d'introduction donnent donc le ton de l'ensemble de l'ouvrage, et sont en tant que telles d'une valeur inestimable.

Guilcher expliquera ensuite l'importance de la consultation des documents anciens pour compléter les enseignements que nous livrent les collectes ethnographiques. Il abordera donc simultanément les fameux livres d'Antonius Arena et de Thoinot Arbeau du XVI ${ }^{\mathrm{e}}$ siècle autant que les danses en chaîne qu'il a pu collecter dans l'Après-Guerre. En décrivant la société rurale d'autrefois, il nous rappellera comment on en est souvent passé de 
la danse chantée, donc sans instrument, aux danses accompagnées par des musiciens. L'évolution menant de la danse en rond à la danse en chaîne ouverte, puis à la contredanse sinon à la danse de couple n'est évidemment pas étrangère à cette nécessité de l'accompagnement musical. Ses réflexions sur les modèles citadins qui ont poussé à l'adoption parfois difficile, sinon au refus, de la contredanse, puis du quadrille, nous rappelle que c'est surtout là que commence notre tradition, et que c'est plutôt la vague des danses tournantes (polka, valse, mazurka...) qui essuieront un refus d'une part de notre population, et du clergé, sinon l'inverse...

Après avoir traité dans le détail des branles (danses rondes), de la farandole et des bourrées, Guilcher clôt avec un dernier chapitre intitulé « variation et tendance ». Il revient alors sur les sujets abordés rapidement lors de son introduction. Cette réflexion, appuyée sur l'observation du terrain, est incontournable pour quiconque veut bien comprendre les ressorts de la tradition orale, en danse certainement, mais aussi dans d'autres domaines. Il résume bien en disant : « [...] la variante ainsi comprise : non une façon nouvelle de traiter un thème préalablement défini, non une liberté prise avec un modèle, un écart par rapport à une norme ; mais une concrétisation éphémère, parmi d'autres également possibles et tout aussi légitimes, d'un dynamisme vivant qui recrée la mélodie et le mouvement à chacune de leurs actualisations. Il faut, cela reconnu, constater que la variation sensu stricto fait à elle seule problème. Ôté le choix délibéré, ôté le souvenir, ôtés l'emprunt au-dehors et l'intervention consciente, il reste un inexpliqué, qui pourrait bien être l'essentiel : une spontanéité créatrice, un surgissement, non calculé, tout dans l'inspiration du moment. Un jaillissement de l'imprévu. »

Sa compréhension de la danse et du danseur, ses interrogations sur ses motivations, conscientes ou inconscientes, sont éclairantes. Lorsqu'il écrit : « les pratiques modernes ne tendent pas automatiquement vers plus de liberté individuelles!», il nous amène à réfléchir sur notre supposée liberté de danseur, que nous considérons comme acquisition récente et par ailleurs souvent absente de la société traditionnelle.

Suite à ce dernier chapitre, on trouve onze annexes allant chacune de deux à dix pages environ, traitant de divers sujets chers à l'auteur.

À la lecture de ce livre, on se met à rêver du jour où nous pourrons faire une telle étude de notre répertoire, et qu'on pourra élucider, en partie tout au moins, des questions importantes relatives à notre tradition dansée au Québec, et dans l'Est du Canada. Par exemple : à quel moment, dans quelles régions, et comment le swing s'est-il ajouté à nos danses de figures ? Étaitil présent avant l'arrivée des danses carrées ? Si oui, dans quelles danses ? Dans le quadrille qui s'enseignait en ville ? Quelles sont les conditions qui ont permis l'avènement de Brandys si richement gigués dans le Saguenay, 
et la conservation des $3 / 2$ associés ? Comment se fait-il que les danseurs de clog soient si populaires dans les années 1920-1930 (en ville à tout le moins) et que nous n'en n'ayons jamais collecté ? Comment expliquer et documenter le style chaloupé des Confitures de Biencourt (dernière partie du quadrille) de cette municipalité située à $65 \mathrm{~km}$ de Trois-Pistoles, à l'intérieur des terres? Quand la gigue fut-elle introduite dans la quatrième partie du quadrille (L'Homme à deux femmes ou Le Veuf) ? Bien que nous ayons collecté de multiples danses québécoises, nous n'avons pas encore abordé ces questions de transformation et d'évolution de notre répertoire dansé, de variations régionales et des variantes permises au sein d'un même groupe.

La profondeur des connaissances de J.-M. Guilcher, et la finesse dont il fait preuve dans son analyse, nous rappelle l'énorme tâche qu'il reste à faire chez nous, principalement dans l'évolution de notre répertoire depuis le XIX ${ }^{\mathrm{e}}$ siècle. Le Québec s'est sans doute trop exclusivement tourné vers l'étude de la Nouvelle-France et de son répertoire, en délaissant du coup le développement culturel au $\mathrm{XIX}^{\mathrm{e}}$ siècle, dont provient la majeure partie de notre répertoire dansé.

Pierre Chartrand

Centre Mnémo, Drummondville

Guillorel, Éva. La Complainte et la plainte. Chanson, justice, cultures en Bretagne (XVI ${ }^{e}$-XVIII ${ }^{e}$ siècles). Rennes, Presses Universitaires de Rennes ; DAstum ; Centre de recherche bretonne et celtique, 2010, 589 p. Ill., cartes + cédérom. ISBN 978-2-7535-1142-2.

La chanson de tradition orale peut-elle être considérée comme source fiable et pertinente pour l'étude historique des sociétés et des cultures à l'époque moderne ? La question est le point de départ pour le magnifique voyage qu'Éva Guillorel nous propose dans La Complainte et la plainte. Chanson, justice, cultures en Bretagne, exploration exhaustive de deux mondes distincts, mais complémentaires : l'un rural et oral, l'autre urbain et lettré. Au cours des 600 pages, ou presque, de grand format qui suivent, l'auteur met en confrontation les représentations de la réalité proposées par les gwerzioù (pluriel de gwerz: complainte tragique de la tradition bretonne, dont la plupart ont été composées du XVI ${ }^{\mathrm{e}}$ au XVIII ${ }^{\mathrm{e}}$ siècle) et les faits rapportés dans archives judiciaires de la même époque. L'exercice confirme sans aucun doute la valeur de cette méthode complémentaire, produisant un tableau vivant et détaillé d'une société et ses conflits, ses croyances et ses coutumes, son imaginaire ainsi que sa réalité concrète.

Éva Guillorel nous présente ici la version remaniée de sa thèse de doctorat 